\title{
Extending INR testing intervals in warfarin patients at a multi-center anticoagulation clinic
}

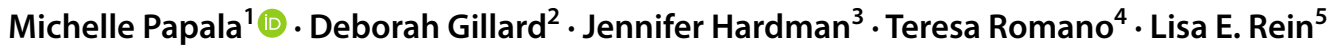

Accepted: 6 September 2021 / Published online: 8 October 2021

() The Author(s), under exclusive licence to Springer Science+Business Media, LLC, part of Springer Nature 2021

\begin{abstract}
Warfarin has been used as an anticoagulant by millions of patients due to its effectiveness, availability, and low cost. Evidence on the safe extension of international normalized ratio (INR) testing frequency remains an area of interest, especially during the recent COVID-19 pandemic. The purpose of this study is to safely extend INR testing intervals in patients throughout a multisite, system-wide anticoagulation clinic. Updates were made to the pharmacist's collaborative practice agreement (CPA) and nurse protocol to optimize practice and allow INR testing interval extension up to a maximum of 8-weeks. The primary outcome was the change in duration between INR tests (INR testing interval) measured before and after providing staff education on clinic updates. The mean duration between INR tests (SD) was 23.69 days (11.29) in the pre-intervention period and 25.58 days (13.91) in the post-intervention period. During the COVID-19 pandemic (post2), intervals were extended further to 27.81 days (14.96), demonstrating a statistically significant increase in INR testing interval from pre-intervention to post-intervention and to post 2 ( $p<0.001$ and $\mathrm{p}<0.001$, respectively). A secondary outcome indicated the mean time in therapeutic range (SD) showed no significant difference in pre-intervention 70.11\% (25.95) versus post-intervention of $69.76 \%(25.69)$ with a difference of $-0.35 \%(29.93)(\mathrm{p}=0.956)$ or versus the post 2 of $68.82 \%(27.20)$ with a difference of $-1.29 \%(33.20)(p=0.120)$. This study showed that changes to the CPA and protocol allowed for a significant increase in INR testing interval while simultaneously maintaining a mean time in therapeutic range $>60 \%$ for the clinic population.
\end{abstract}

Keywords Warfarin · INR testing interval · Time in therapeutic range (TTR) · Protocol · Collaborative practice agreement · COVID-19 pandemic

\section{Highlights}

Michelle Papala

michelle.papala@froedtert.com

1 Froedtert \& The Medical College of Wisconsin, Froedtert Hospital, 9200 W. Wisconsin Ave., Milwaukee, WI 53226, USA

2 Anticoagulation Clinic, Froedtert \& The Medical College of Wisconsin, Froedtert Hospital, 9200 W. Wisconsin Ave., Milwaukee, WI 53226, USA

3 Ambulatory Pharmacy Services, Froedtert \& The Medical College of Wisconsin, Froedtert Hospital, 9200 W. Wisconsin Ave., Milwaukee, WI 53226, USA

4 Anticoagulation and Rheumatology Clinic, Froedtert \& The Medical College of Wisconsin, Froedtert Hospital, 9200 W. Wisconsin Ave., Milwaukee, WI 53226, USA

5 Division of Biostatistics, Institute for Health \& Equity, Medical College of Wisconsin, 8701 Watertown Plank Rd, Milwaukee, WI 53226, USA
- Changes to the nurse protocol and the pharmacist's collaborative practice agreement (CPA), accompanied by education, resulted in a significant extension of the INR testing interval in the study participants.

- Increase in INR testing interval length did not significantly decrease overall clinic mean time in therapeutic range (TTR).

- During the COVID-19 pandemic, INR testing intervals were further increased to accommodate the national quarantine and safer at home orders without reducing the clinic's mean TTR.

- As a result of this project, the nurse protocol was also updated to align with the 8-week testing interval allowed in the pharmacist CPA. Other implications for future directions include exploring the safety of extending INR testing intervals up to 12 -weeks. 


\section{Introduction}

Warfarin has been used as an anticoagulant by millions of patients across the world due to its effectiveness, widespread availability, and low cost [1,2]. For decades, warfarin has been prescribed to prevent and treat thromboembolic events [1]. However, there are challenges to its safe utilization, such as its narrow therapeutic window, variable biological effects, and potential for drug and food interactions $[3,4]$. To achieve desirable outcomes and optimize the benefit versus risk of warfarin therapy, monitoring of patient's International Normalized Ratio (INR) and adjusting the warfarin dose to maintain the INR in the desired goal range is essential [5, 6]. Furthermore, optimizing how often INR values are within therapeutic range, also known as time in therapeutic range (TTR), can improve outcomes, as an increased TTR is associated with a reduction in adverse bleeding and thromboembolic events $[5,6]$.

Many different methodologies exist for calculating TTR. The Rosendaal method is used most commonly because it incorporates time [6-8]. The Rosendaal method, also known as linear interpolation, draws a line from one measured INR to the next measured INR. The days between these two points are assigned INR values, assuming that changes between consecutive measurements are linear over time. TTR is calculated by averaging measured or assigned value for all days in a selected period of time [9]. Major recent clinical trials have found most anticoagulation clinics (ACC) across the country to achieve a mean TTR value in the mid-to-high $60 \%$ range [9]. Specifically, a retrospective cross-sectional study of patients with atrial fibrillation stratified subjects according to TTR and determined the following subgroups: TTR $<60 \%$ (poor anticoagulation control); TTR 60-75\% (good anticoagulation control); TTR $>75 \%$ (excellent anticoagulation control) [8]. Clinics who specialize in anticoagulation should strive to continually advance and achieve excellent anticoagulation control for their population.

Froedtert \& the Medical College of Wisconsin's (F\&MCW) ACC utilizes a multidisciplinary approach to managing warfarin patients with a collaborative practice agreement (CPA) and a nurse protocol for pharmacists and nurses, respectively. The clinic manages approximately 4000 patients across the F\&MCW enterprise who are on warfarin. Patients are managed in a variety of ways: inperson visit with point of care INR testing or telephone call following a venous lab draw, home health draw, or self-test. F\&MCW has four clinic sites available for inperson visits.

Monitoring of patients while on warfarin is a critical component of safe and efficacious therapy. The American College of Chest Physicians Antithrombotic Therapy and
Prevention of Thrombosis 2012 guidelines indicate a level 2B rating for an INR testing frequency of up to 12-weeks in patients on warfarin who have been stable for at least 3 months. A $2 \mathrm{~B}$ rating indicates a weak recommendation based on moderate quality evidence [10]. While further evidence would be beneficial to strengthen recommendations for extending INR testing intervals to 8 - or 12-week frequency, available data suggests no increased risk for bleeding or thromboembolism when extending INRs up to 8 -weeks in stable patients [11-13].

With the start of the COVID-19 pandemic, many clinicians were required to extend INR testing intervals as safely as possible. Patients who take warfarin often have medical conditions that place them at high risk for severe COVID-19 disease severity and are often older in age which according to the Centers for Disease Control and Prevention, also places individuals at increased risk for severe illness including hospitalization, intensive care admissions, and death [14-18]. Compared to younger adults, individuals $65-74$ years old are 5 times more likely to be hospitalized and 90 time more likely to die if they have COVID-19 infection [18-21]. Patients afraid of infection may be hesitant or refuse to come in for appointments and lab work even if considered essential. One way to help ease patient hesitation include testing options that do not require them to enter a hospital or clinic such as drive-up INR testing $[22,23]$. Another precaution is to ensure INR testing intervals are extended when clinically appropriate. Clinician decision making is used to ensure patients are clinically appropriate if they are adherent with stable INRs and no new interacting medications, bleeding or thrombotic episodes.

It was determined that opportunities existed to optimize patient management within F\&MCW ACC's CPA and protocol to allow INR testing interval to be extended when clinically appropriate. Prior to the implementation of this study, F\&MCW ACC allowed for INR testing intervals to be extended up to 6-weeks based on the ACC clinician's discretion and up to 8-weeks with the referring provider's approval. Neither the CPA nor protocol included recommendations for when and how to extend INR testing intervals beyond 6 weeks, and there is limited published literature to provide guidance. This led to limited or delayed extension of INR testing intervals for most F\&MCW ACC patients. The objective of this study was to safely extend INR testing intervals, up to a maximum of 8-weeks, in clinically eligible patients. This was done by updating the CPA and nurse protocol and then providing staff with education on these updates. Education consisted of a presentation summarizing the clinic's approach to extending INRs and workflow changes followed by case-based practice questions. All staff were required to attend either an in-person or virtual education session. 


\section{Methods}

The study was performed at a multisite, single enterprise anticoagulation service at $\mathrm{F} \& \mathrm{MCW}$. Updates were made to the pharmacist CPA to optimize practice and allow INR testing interval extension up to a maximum of 8-weeks. The nurse protocol underwent updates but remained at a maximum testing interval of 6-weeks unless approved by the overseeing pharmacist to extend to 8-weeks. Protocol and CPA updates along with clinic team education was considered the study intervention. This pre-post observational cohort study analyzed data for 3 months prior to the intervention (pre-), for 3-months post intervention (post-), and 6-months post-intervention (post2). The second post-data collection point, or the post2, was approved by the research committee separately from the initial 3-month follow-up data collection to determine COVID-19 pandemic's impact on the effects of the intervention. Patients served as their own control group. Descriptive statistics were used to summarize characteristics of the study cohort. This study was deemed a quality improvement project by an independent local Institutional Review Board (IRB); therefore, this project was exempt from IRB approval.

\section{Selection and description of participants}

This study analyzed outcomes from data reports pulled on December 2, 2019, March 2, 2020, and June 2, 2020. For each report, data were pulled from a single point of time; roughly 3 months prior to the intervention, 3-months post-intervention, and 6-months post-intervention (post2) (Fig. 1). Since the system's electronic health record is set to compute TTR over a 3-month time range, this reporting interval was utilized to help prevent bias by preventing overlap of TTR from the pre- and post-intervention periods. Patients were eligible to be included if they were $>18$ years old, managed by F\&MCW ACC, on warfarin anticoagulation therapy for at least three months, and managed with a standard INR goal range of 2-3 or 2.5-3.5. Patients were excluded if they had a Ventricular Assist Device (VAD) or self-testing meter since these patients perform more frequent INR testing, had a planned duration of warfarin of 6 months or less, or did not have at least one visit during the pre- and post-intervention periods. All other patients, even those not likely to be clinically appropriate for interval extension, were included.

\section{Study outcomes}

The study's primary outcome was the change in duration between INR tests (INR testing interval) as calculated by the time between INR draw and recommended follow-up date in the overall study population measured before and after the study intervention. The most recent INR testing interval between INR draw and recommended follow-up date on the day the reports were run was used for each patient. Secondary outcomes included: TTR measured in the pre-, post- and post 2 periods and comparison of INR testing interval based on visit type (i.e. office visit or telephone visit). In addition to reporting INR testing in number of days, a post hoc analysis was completed to compare INR extension based on grouped time intervals ranges.

\section{Statistical analysis}

Our statistical review determined that we would need at least 1053 patients to detect the effect of at least 0.1 days between the average INR testing intervals pre- and post- intervention with $90 \%$ power.

Study variables were summarized by the mean, standard deviation, median, quartiles, and range for continuous variables and frequency and percentage for categorical variables. INR testing interval and TTR were each compared between pre- and post-intervention periods using a Wilcoxon signed-rank test. INR testing interval was compared between pre- and post-periods within each encounter type subgroup (office visits or telephone visits) using a Wilcoxon signedrank test; the differences in INR testing interval (post to pre, post-2 to post, post-2 to pre) was compared between encounter types using a Wilcoxon rank-sum test.

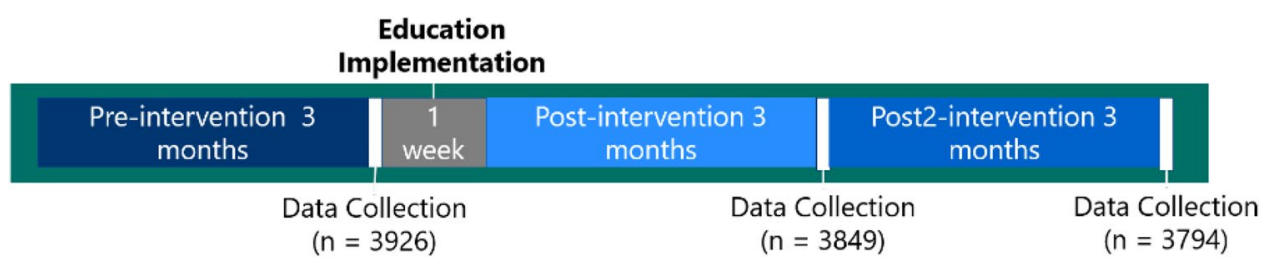

Fig. 1 Timeline of project. Shown is the timeline of project events including the pre-, post-, and post2-intervention time frames in relation to data collection time points and educational interventions. The sample size of participants from each time frame is included. A total of 3392 patients had data in all 3 time periods and were included in the analysis 
INR testing intervals were also categorized into 5 groups: $<2,2-3,4-5,6-7$, and $8+$ weeks. INR testing interval categories were compared between pre-, postand post2-intervention periods using a nominal symmetry test and a weighted Kappa test. Patients were further categorized as moving to a lower INR testing interval category, moving to a higher INR testing interval category, or staying in the same category from the pre- and to postintervention periods; a sign test was used to test whether patients tended to stay in the same category or transition.

All statistical analyses were performed using $\mathrm{R}$ version 3.6.0 (2019-04-26) (R Foundation for Statistical Computing, http://www.R-project.org). All tests were two-sided and $p<0.05$ was considered statistically significant. All analyses employed an "available-case" approach to missing data. No adjustments were made for multiple testing.

Table 1 Baseline characteristic

\begin{tabular}{ll}
\hline Age (years) & \\
Mean (SD) & $72.21(13.63)$ \\
Median [Q1, Q3] & $73.63[64.64,82.15]$ \\
Min, max & $18.03,101.93$ \\
Gender [n (\%)] & \\
Male & $1901(56.0 \%)$ \\
Female & $1491(44.0 \%)$ \\
\hline
\end{tabular}

\section{Results}

\section{Baseline characteristics}

The extracted data includes 3926 patients in the pre-, 3849 in the post-, and 3794 in the post2-intervention. Of these patients, a total of 3392 patients met criteria and had data in all 3 time periods and were included in the analysis. The study population had a mean age of 73.6 years and was comprised of slightly more female than male subjects, 56 and $44 \%$ respectively (Table 1 ).

\section{Primary outcome}

The mean INR testing interval (SD) was 23.69 days (11.29) in the pre- and 25.58 days (13.91) in the post-intervention period. This resulted in a significant difference of 1.90 days $(\mathrm{p}<0.001)$ from the pre- to post-intervention period (Fig. 2). The mean INR testing interval (SD) was 27.81 days (14.96) in the post2. A significant difference was also seen from the post- to post 2 intervals of 2.23 days $(p<0.001)$ and in the pre- to post 2 - intervention of 4.12 days $(\mathrm{p}<0.001)$.

\section{Secondary outcomes}

The mean TTR (SD) measured pre- and post-intervention showed no significant difference in pre- $70.11 \%$ (25.95) versus post-intervention of $69.76 \%$ (25.69) with a difference of $-0.35 \%(29.93)(p=0.956)$. In the post 2 , the mean TTR
Fig. 2 Primary outcome. The box and whisker plot demonstrates the outcomes of INR intervals seen for the pre-, post-, and post2-intervention data collection points

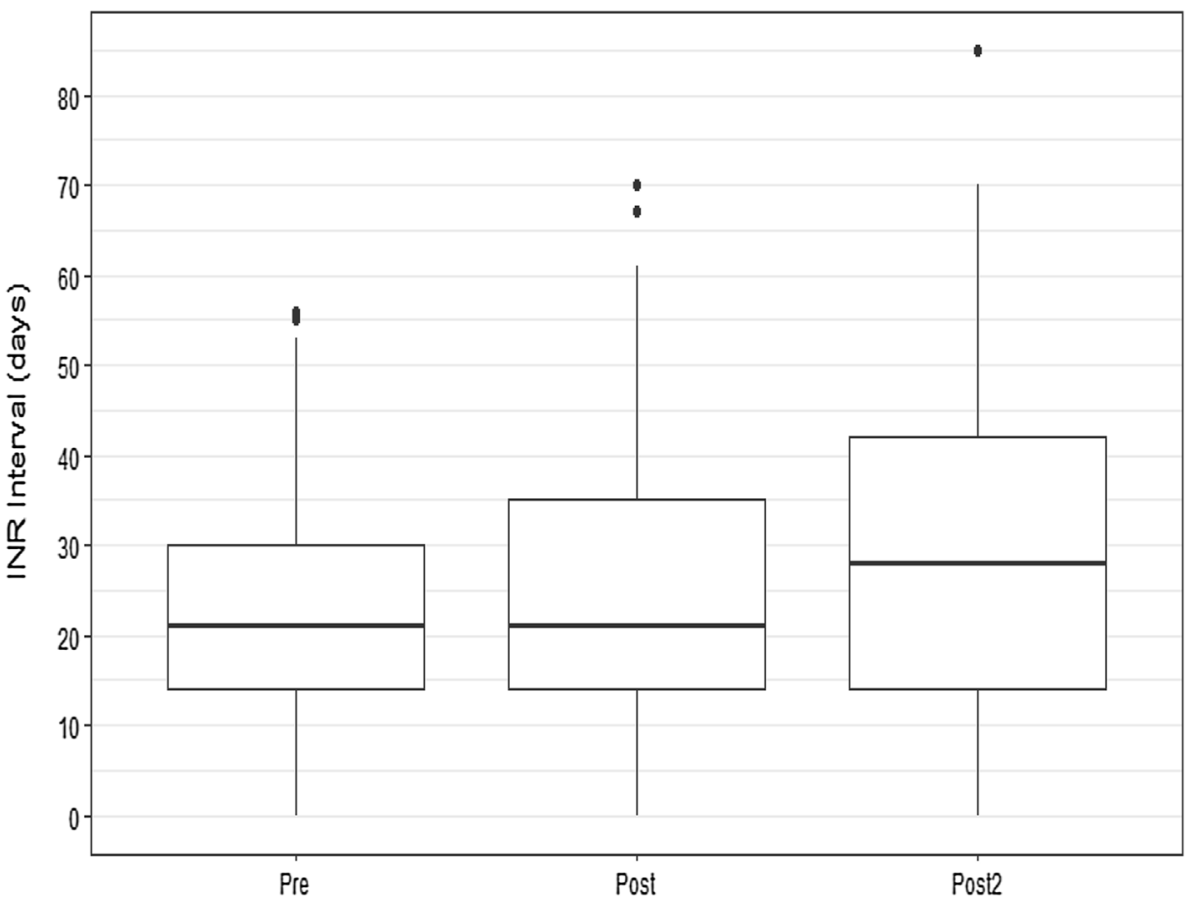


(SD) was $68.82 \%$ (27.20). There was a non-significant difference in TTR between the post- and the post $2(p=0.354)$ and from the pre- to post $2(\mathrm{p}=0.120)$.

Comparison of INR testing frequency based on visit type (office visit or telephone visit) revealed a significant increase in INR testing interval for each group post-intervention in individuals with available data. Office visits resulted in a mean INR testing interval (SD) of 28.54 days (10.44) in the pre- compared with 30.76 days (13.43) in the post-intervention; a difference of 2.22 days $(12.13)(\mathrm{p}<0.001)$. The mean INR testing interval was 34.13 days (13.82) for the office visits in the post 2 ; a mean difference of 5.70 days $(p<0.001)$ compared to the pre-intervention period. While the INR testing interval increased in both visit types, the office visit type resulted in a greater extension in mean INR testing interval in the pre to post 2 than the telephone calls (5.70 vs. 3.64 days, respectively) (Table 2).

Data on INR testing interval extension was broken down in a post hoc analysis to look at INR testing interval category change and showed that $41.5 \%$ of patients moved to a longer INR testing interval from pre- to post 2 groups, while $36.4 \%$ stayed in the same interval category and $22.2 \%$ moved to a shorter interval category. The change in patients extending into the 8-week interval group was statistically significant, with 3 patients pre-, 236 patients post- and 357 patients post $2(\mathrm{p}<0.001)$.

\section{Discussion}

This study showed that pharmacist CPA and nurse protocol changes followed by staff education allowed for a significant increase in the mean INR testing interval of 1.90 days $(\mathrm{p}<0.001)$ from the pre- to the post-intervention and 4.12 days $(\mathrm{p}<0.001)$ from the pre- to post2. Time in therapeutic range was utilized as a surrogate safety measure and showed that an INR testing interval extension to 8-weeks was safe, as there was no significant decrease in overall population TTR. Thus, ACC was able to extend testing intervals while maintaining TTR, similar to recommendations provided by Chest Guidelines and other published literature. While the extension of INR testing intervals by this amount may not seem substantial, this change had significant impacts on workload in the clinic. With the mean change from 23.69 days pre- to 27.81 days post 2 , there was an average of 2.28 fewer tests per year per patient. Multiplied by the study population size of 3392 patients, this results in about 7733 fewer tests managed by the clinic over the course of a year or about 30 fewer results each weekday.

The study had some limitations. First, INR is a measurement that varies, as patients who undergo a procedure, have changes to their medications, or experience illnesses may experience out-of-range results [1, 3, 4]. This may cause lowering of overall TTR but would be independent of the change in TTR caused by extending INR testing intervals in a specific timeframe (i.e. 3 months) $[4,5]$. It is difficult to control for these variables and is an inherent limitation of this study. Another limitation is that there were no data collected on the correlation between INR testing interval extension and the effect of bleeding and thrombotic events. The complexity of warfarin dosing makes tracking and relating bleeding or thrombotic events to INR testing intervals difficult and is outside the scope of this study. Finally, the INR testing intervals were calculated using the difference between the current INR test date and the recommended return date. This likely underestimates the actual INR testing interval as many patients returned to clinic later than the recommended date, especially during post 2 .

Despite the limitations, this study showed effectiveness and safety of an education intervention with changes to the pharmacist CPA and nurse protocol at the F\&MCW ACC. Given the unexpected COVID-19 pandemic, pharmacists and nurses needed to continue to evaluate the INR testing interval weighing the possible risk of COVID exposure when getting INR tested with the risk of over extending the testing interval.

After successful completion of this study, the F\&MCW ACC updated the nurse protocol to align with the pharmacist CPA to allow the 8-week testing interval. Other future directions may include considering guidance in ACCP Chest Guidelines to update the CPA to allow INR testing intervals up to 12 -weeks. 
Table 2 Mean INR testing interval change by visit type

\begin{tabular}{|c|c|c|c|c|c|c|c|c|c|}
\hline & \multicolumn{4}{|c|}{ Pre office visit/post office visit $(n=1319)$} & \multicolumn{4}{|c|}{ Pre telephone/post telephone $(\mathrm{n}=1879)$} & \multirow{2}{*}{$\begin{array}{l}\text { Comparison } \\
\text { of diff's } \\
\text { p-value }\end{array}$} \\
\hline & Pre & Post & Diff & $\begin{array}{l}\text { Paired } \\
\text { p-value }\end{array}$ & Pre & Post & Diff & $\begin{array}{l}\text { Paired } \\
\text { p-value }\end{array}$ & \\
\hline $\begin{array}{l}\text { INR testing } \\
\text { interval } \\
\text { (days) }\end{array}$ & & & & $<0.001$ & & & & $<0.001$ & 0.125 \\
\hline Mean (SD) & $\begin{array}{l}28.54 \\
\quad(10.44)\end{array}$ & $\begin{array}{l}30.76 \\
\quad(13.43)\end{array}$ & $2.22(12.13)$ & & $\begin{array}{l}20.53 \\
\quad(10.61)\end{array}$ & $\begin{array}{l}22.43 \\
\quad(13.28)\end{array}$ & $1.90(11.15)$ & & \\
\hline \multirow[t]{3}{*}{$\begin{array}{c}\text { Median } \\
{[\min ,} \\
\max ]\end{array}$} & $\begin{array}{l}28.00[3.00 \\
56.00]\end{array}$ & $\begin{array}{c}28.00[3.00 \\
70.00]\end{array}$ & $\begin{array}{l}1.00 \\
{[-42.00} \\
49.00]\end{array}$ & & $\begin{array}{l}14.00[0.00 \\
55.00]\end{array}$ & $\begin{array}{c}15.00[0.00 \\
67.00]\end{array}$ & $\begin{array}{l}0.00 \\
{[-38.00} \\
43.00]\end{array}$ & & \\
\hline & \multicolumn{4}{|c|}{ Post office visit/post COVID office visit $(n=1239)$} & \multicolumn{4}{|c|}{ Post telephone/post COVID telephone $(\mathrm{n}=1913)$} & $\begin{array}{l}\text { Comparison } \\
\text { of diff's }\end{array}$ \\
\hline & Post & Post2 & Diff & $\begin{array}{l}\text { Paired } \\
\text { p-value }\end{array}$ & Post & Post2 & Diff & $\begin{array}{l}\text { Paired } \\
\text { p-value }\end{array}$ & p-value \\
\hline $\begin{array}{l}\text { INR testing } \\
\text { interval } \\
\text { (days) }\end{array}$ & & & & $<0.001$ & & & & $<0.001$ & $<0.001$ \\
\hline Mean (SD) & $\begin{array}{l}30.83 \\
(13.49)\end{array}$ & $\begin{array}{l}34.13 \\
\quad(13.82)\end{array}$ & $3.29(13.36)$ & & $\begin{array}{l}22.30 \\
\quad(13.27)\end{array}$ & $\begin{array}{l}24.09 \\
\quad(14.34)\end{array}$ & $1.79(12.47)$ & & \\
\hline \multirow[t]{3}{*}{$\begin{array}{c}\text { Median } \\
{[\min ,} \\
\max ]\end{array}$} & $\begin{array}{c}28.00[3.00, \\
70.00]\end{array}$ & $\begin{array}{l}30.00[4.00, \\
85.00]\end{array}$ & $\begin{array}{l}2.00 \\
{[-50.00} \\
42.00]\end{array}$ & & $\begin{array}{c}15.00[0.00 \\
67.00]\end{array}$ & $\begin{array}{l}20.00[1.00, \\
64.00]\end{array}$ & $\begin{array}{l}0.00 \\
{[-42.00} \\
52.00]\end{array}$ & & \\
\hline & \multicolumn{4}{|c|}{ Pre office visit/post COVID office visit $(n=1234)$} & \multicolumn{4}{|c|}{ Pre telephone/post COVID telephone $(\mathrm{n}=1868)$} & $\begin{array}{l}\text { Comparison } \\
\text { of diff's }\end{array}$ \\
\hline & Pre & Post2 & Diff & $\begin{array}{l}\text { Paired } \\
\text { p-value }\end{array}$ & Pre & Post2 & Diff & $\begin{array}{l}\text { Paired } \\
\text { p-value }\end{array}$ & p-value \\
\hline $\begin{array}{l}\text { INR testing } \\
\text { interval } \\
\text { (days) }\end{array}$ & & & & $<0.001$ & & & & $<0.001$ & $<0.001$ \\
\hline Mean (SD) & $\begin{array}{l}28.55 \\
\quad(10.44)\end{array}$ & $\begin{array}{l}34.25 \\
\quad(13.74)\end{array}$ & $5.70(13.90)$ & & $\begin{array}{l}20.54 \\
\quad(10.64)\end{array}$ & $\begin{array}{l}24.17 \\
\quad(14.34)\end{array}$ & 3.64 (12.67) & & \\
\hline $\begin{array}{c}\text { Median } \\
{[\min ,} \\
\max ]\end{array}$ & $\begin{array}{l}28.00[3.00 \\
56.00]\end{array}$ & $\begin{array}{l}30.00[4.00 \\
85.00]\end{array}$ & $\begin{array}{l}7.00 \\
{[-49.00} \\
49.00]\end{array}$ & & $\begin{array}{c}14.00[0.00 \\
55.00]\end{array}$ & $\begin{array}{l}20.00[1.00 \\
64.00]\end{array}$ & $\begin{array}{l}0.00 \\
\quad[-37.00 \\
53.00]\end{array}$ & & \\
\hline
\end{tabular}

Author contributions All authors contributed to the study conception and design. The first draft of the manuscript was written by MP and TR and all authors commented on previous versions of the manuscript. All authors read and approved the final manuscript.

Funding No funding was received for conducting this study.

\section{Declarations}

Conflict of interest The authors have no relevant financial or non-financial interests to disclose.

Ethical approval This is a quality improvement study. Updates to CPA and nurse protocol were performed by clinic staff members under the usual practice of document updates and would have occurred independent of this study. All documents were approved through standard institutional approval procedures. The Froedtert \& Medical College of
Wisconsin Pharmacy Research Ethics Committee has confirmed that no ethical approval is required.

\section{References}

1. Pirmohamed M (2006) Warfarin: almost 60 years old and still causing problems. Br J Clin Pharmacol 62(5):509-511. https:// doi.org/10.1111/j.1365-2125.2006.02806.x

2. Farsad BF, Abbasinazari M, Dabagh A, Bakshandeh H (2016) Evaluation of time in therapeutic range (TTR) in patients with non-valvular atrial fibrillation receiving treatment with warfarin in Tehran, Iran: a cross-sectional study. J Clin Diagn Res JCDR 10(9):FC04-FC06. https://doi.org/10.7860/JCDR/2016/21955. 8457

3. Ageno W, Gallus AS, Wittkowsky A, Crowther M, Hylek EM, Palareti G (2012) Oral anticoagulant therapy: antithrombotic 
therapy and prevention of thrombosis, 9th ed: American College of Chest Physicians Evidence-Based Clinical Practice Guidelines. Pract Guidel Chest 141(2 Suppl):e44S-e88S. https://doi. org/10.1378/chest.11-2292

4. Ansell J, Jacobson A, Levy J, Völler H, Hasenkam JM, International Self-Monitoring Association for Oral Anticoagulation (2005) Guidelines for implementation of patient self-testing and patient self-management of oral anticoagulation. International consensus guidelines prepared by International Self-Monitoring Association for Oral Anticoagulation. Int J Cardiol 99(1):37-45. https://doi.org/10.1016/j.ijcard.2003.11.008

5. Schmitt L, Speckman J, Ansell J (2003) Quality assessment of anticoagulation dose management: comparative evaluation of measures of time-in-therapeutic range. J Thromb Thrombolysis 15(3):213-216. https://doi.org/10.1023/B:THRO.0000011377. 78585

6. Farsad BF, Abbasinazari M, Dabagh A, Bakshandeh H (2016) Evaluation of time in therapeutic range (TTR) in patients with non-valvular atrial fibrillation receiving treatment with warfarin in Tehran, Iran: a cross-sectional study. J Clin Diagn Res JCDR 10(9):FC04-FC06. https://doi.org/10.7860/JCDR/2016/21955. 8457

7. Azar AJ, Deckers JW, Rosendaal FR, van Bergen PF, van der Meer FJ, Jonker JJ, Briët E (1994) Assessment of therapeutic quality control in a long-term anticoagulant trial in post-myocardial infarction patients. Thromb Haemost 72(3):347-351

8. Melamed OC, Horowitz G, Elhayany A, Vinker S (2011) Quality of anticoagulation control among patients with atrial fibrillation. Am J Manag Care 17(3):232-237

9. Reiffel JA (2017) Time in the therapeutic range (TTR): an overly simplified conundrum. J Innov Card Rhythm Manag 8(3):26432646. https://doi.org/10.19102/icrm.2017.080302

10. Holbrook A, Schulman S, Witt DM, Vandvik PO, Fish J, Kovacs MJ, Svensson PJ, Veenstra DL, Crowther M, Guyatt GH (2012) Evidence-based management of anticoagulant therapy: antithrombotic therapy and prevention of thrombosis, 9th ed: American College of Chest Physicians Evidence-Based Clinical Practice Guidelines. Chest 141(2 Suppl):e152S-e184S. https://doi.org/ 10.1378/chest.11-2295

11. Witt DM, Delate T, Clark NP, Martell C, Tran T, Crowther MA, Garcia DA, Ageno W, Hylek EM, Warped Consortium (2010) Twelve-month outcomes and predictors of very stable INR control in prevalent warfarin users. J Thromb Haemost JTH 8(4):744-749. https://doi.org/10.1111/j.1538-7836.2010.03756.x

12. Witt DM, Delate T, Clark NP, Martell C, Tran T, Crowther MA, Garcia DA, Ageno W, Hylek EM, Warfarin Associated Research Projects and other EnDeavors (WARPED) Consortium (2009) Outcomes and predictors of very stable INR control during chronic anticoagulation therapy. Blood 114(5):952-956. https:// doi.org/10.1182/blood-2009-02-207928
13. Rose AJ, Ozonoff A, Henault LE, Hylek EM (2008) Warfarin for atrial fibrillation in community-based practise. J Thromb Haemost JTH 6(10):1647-1654. https://doi.org/10.1111/j.1538-7836.2008. 03075.x

14. Centers for Disease Control and Prevention (2021) People with certain medical conditions. https://www.cdc.gov/coronavirus/ 2019-ncov/need-extra-precautions/people-with-medical-condi tions.html?CDC_AA_refVal=https $\% 3 \mathrm{~A} \% 2 \mathrm{~F} \% 2 \mathrm{Fwww} . \mathrm{cdc}$. gov $\%$ 2Fcoronavirus\%2F2019-ncov\%2Fneed-extra-precautions\%2Fgro ups-at-higher-risk.html\#heart-conditions. Accessed 5 Febr 2021

15. Zheng Z, Peng F, Xu B, Zhao J, Liu H, Peng J, Li Q, Jiang C, Zhou Y, Liu S, Ye C, Zhang P, Xing Y, Guo H, Tang W (2020) Risk factors of critical \& mortal COVID-19 cases: a systematic literature review and meta-analysis. J Infect 81(2):e16-e25. https://doi.org/ 10.1016/j.jinf.2020.04.021

16. Li B, Yang J, Zhao F, Zhi L, Wang X, Liu L, Bi Z, Zhao Y (2020) Prevalence and impact of cardiovascular metabolic diseases on COVID-19 in China. Clin Res Cardiol Off J Ger Card Soc 109(5):531-538

17. Zhao Q, Meng M, Kumar R, Wu Y, Huang J, Lian N, Deng Y, Lin S (2020) The impact of COPD and smoking history on the severity of COVID-19: a systemic review and meta-analysis. J Med Virol 92(10):1915-1921. https://doi.org/10.1002/jmv.25889

18. Centers for Disease Control and Prevention (2020) Older adults at greater risk of requiring hospitalization or dying if diagnosed with COVID-19. https://www.cdc.gov/coronavirus/2019-ncov/ need-extra-precautions/older-adults.html Accessed 5 Febr 2021

19. Shahid Z, Kalayanamitra R, McClafferty B, Kepko D, Ramgobin D, Patel R, Aggarwal CS, Vunnam R, Sahu N, Bhatt D, Jones K, Golamari R, Jain R (2020) COVID-19 and older adults: what we know. J Am Geriatr Soc 68(5):926-929. https://doi.org/10.1111/ jgs. 16472

20. Jayaweera M, Perera H, Gunawardana B, Manatunge J (2020) Transmission of COVID-19 virus by droplets and aerosols: a critical review on the unresolved dichotomy. Environ Res 188:109819. https://doi.org/10.1016/j.envres.2020.109819

21. Centers for Disease Control and Prevention (2020) Coronavirus Disease 2019 (COVID-19): how COVID-19 spreads. https:// www.cdc.gov/coronavirus/2019-ncov/prepare/transmission.html. Accessed 5 Febr 2021

22. Truong L, Whitfield K, Nickerson-Troy J, Francoforte K (2021) Drive-thru anticoagulation clinic: can we supersize your care today? J Am Pharm Assoc JAPhA 61(2):e65-e67. https://doi.org/ 10.1016/j.japh.2020.10.016

23. Ostroff J, Roggie S, Opitz B (2020) Curbside Coumadin Clinics offer testing during COVID-19 crisis. J Pharm Pract 33(6):734735. https://doi.org/10.1177/0897190020944054

Publisher's Note Springer Nature remains neutral with regard to jurisdictional claims in published maps and institutional affiliations. 